\title{
Stereotactic Radiosurgery Alone for One to Two Brain Metastases from Cancer of Unknown Primary
}

\author{
LIESA DZIGGEL ${ }^{1}$, AMIRA BAJROVIC ${ }^{2}$, STEVEN E. SCHILD $^{3}$ and DIRK RADES ${ }^{1}$ \\ ${ }^{1}$ Department of Radiation Oncology, University of Lübeck, Lübeck, Germany; \\ ${ }^{2}$ Department of Radiotherapy, University Medical Center Eppendorf, Hamburg, Germany; \\ ${ }^{3}$ Department of Radiation Oncology, Mayo Clinic, Scottsdale, AZ, U.S.A.
}

\begin{abstract}
Background/Aim: The use of stereotactic radiosurgery (SRS) alone has become popular for treating patients with a limited number of brain metastases. In very few patients, the primary tumor leading to cerebral spread is unknown. This study investigated the role of SRS for this rare situation. Patients and Methods: Eight patients with 1-2 brain metastases from cancer of unknown primary (CUP) received SRS alone (median dose $20 \mathrm{~Gy}$ ). Five clinical factors were evaluated for association with local control of the irradiated lesions, freedom from new cerebral lesions and survival. Results: Six-month and 12-month survival rates were $63 \%$ and $63 \%$, respectively. Improved survival was associated with male gender and only one cerebral lesion. Local control rates at 6 and 12 months were $100 \%$. Six-month rate of freedom from new cerebral lesions was 86\%. Conclusion: SRS appeared effective and resulted in promising local control and survival rates in patients with 1-2 brain metastases from CUP.
\end{abstract}

Treatment with stereotactic radiosurgery (STS) alone instead of SRS plus whole-brain irradiation for patients with very few brain metastases has become more popular in order to reduce the risk of radiation-related side-effects (1). Randomized studies have shown that the addition of wholebrain irradiation to SRS can reduce the rate of new brain metastases outside the areas treated with SRS. Unfortunately, the addition of whole-brain irradiation also results in significantly higher rates of neuro-cognitive dysfunction $(2$,

Correspondence to: Professor Dirk Rades, MD, Department of Radiation Oncology, University of Lübeck, Lübeck, Ratzeburger Allee 160, 23538 Lübeck, Germany. Tel: +49 45150045401, Fax: +49 45150045404, e-mail: rades.dirk@gmx.net

Key Words: Cancer of unknown primary, brain metastases, stereotactic radiosurgery, local control, freedom from new cerebral lesions, survival.
3). However, since new cerebral metastases can also impair the patient's neuro-cognitive function, the most appropriate treatment regimen for those with a very limited number of cerebral lesions is controversial. The most appropriate treatment also depends on the type of primary tumor, its radiosensitivity, the probability of development of multiple cerebral lesions following SRS alone, and the patient's expected survival time (1).

If the primary tumor that metastasized to the brain is unknown, the decision to use SRS alone or a combination of SRS and whole-brain irradiation is even more difficult. Patients with very few brain metastases from a cancer of unknown primary (CUP) are quite rare (4). Many of them have a poor survival prognosis and may not live long enough to experience new brain metastases following SRS alone, or progression of the lesions treated with SRS. Neuro-cognitive deficits caused by whole-brain irradiation can occur within a few months of treatment and may have a negative impact on the patient's quality of life $(2,3)$. Thus, it appears reasonable to use SRS alone in these patients. However, only very few data are available for this particular situation. Therefore, the present study was performed to investigate the effect of SRS alone for patients with one to two cerebral metastases from cancer of unknown primary.

\section{Patients and Methods}

The data of eight patients (Table I) who were treated with SRS alone for one to two brain metastases from CUP were retrospectively analyzed for local control of the irradiated lesions, freedom from new cerebral lesions, and survival. In addition, the potential impact of five clinical factors was investigated. These factors were age $(\leq 61$ $v s .>61$ years), gender, number of cerebral lesions (one vs. two), presence of extracranial metastasis (no vs. yes) and recursive partitioning analysis (RPA) class ( 1 vs. 2) (5). Patients belonging to RPA class 1 had a Karnofsky performance score of $\geq 70$ or more, a controlled primary tumor, no extracranial metastases and were aged 64 years or younger. RPA class 2 patients also had a Karnofsky score of $\geq 70$ but did not have all three other favorable characteristics. Local control of the irradiated lesions, freedom from new cerebral 
Table I. Clinical factors evaluated for treatment outcomes.

\begin{tabular}{lc}
\hline Factor & Number of patients \\
\hline Age & \\
$\leq 61$ Years & 4 \\
$>61$ Years & 4 \\
Gender & \\
Female & 4 \\
$\quad$ Male & 4 \\
Number of cerebral lesions & 5 \\
1 & 3 \\
2 & \\
Extracranial metastasis & 4 \\
No & 4 \\
Yes & \\
Recursive partitioning analysis class & 3 \\
Class 1 & 5 \\
Class 2 & \\
\hline
\end{tabular}

lesions and survival were references from the day of SRS and calculated with the Kaplan-Meier method (6). Those clinical factors achieving significance $(p<0.05)$ on univariate analysis $($ log-rank) were subsequently analyzed in a multivariate manner using a Cox regression model.

\section{Results}

For the entire cohort, the 6- and 12-month actuarial survival rates were $63 \%$ and $63 \%$, respectively (Table II). Median survival time was not reached during the period of followup. On univariate analysis, improved survival was associated with male gender $(p=0.040)$ and presence of only one cerebral lesion $(p=0.040)$. On subsequent Cox regression analysis, a trend was found for both gender $(p=0.096)$ and number of cerebral lesions $(p=0.096)$.

Recurrence within the irradiated areas of the brain was not observed in any of the patients. Thus, the local control rates were $100 \%$ at both 6 and 12 months. New cerebral lesions outside the irradiated areas were observed in two patients after 2, and 7 months, respectively. The rate of freedom from new cerebral lesions at 6 months was $86 \%$ (Table III). The corresponding rate at 12 months was not available. However, the rate of freedom from new cerebral lesions at 10 months was $64 \%$. None of the investigated clinical factors reached significance with respect to any association with freedom from new cerebral lesions.

\section{Discussion}

Brain metastases develop in about one-third of patients with cancer (4). The most common primary tumor types resulting in brain metastases are lung and breast cancer. In a small proportion of patients (fewer than 1\%) with cerebral
Table II. Analysis of survival.

\begin{tabular}{lcc}
\hline Factor & At 6 months & $p$-Value \\
\hline Age & & \\
$\leq 61$ Years & 75 & 0.62 \\
$>61$ Years & 50 & \\
Gender & & \\
Female & 25 & $\mathbf{0 . 0 4 0}$ \\
Male & 100 & \\
Number of cerebral lesions & & \\
1 & 100 & $\mathbf{0 . 0 4 0}$ \\
2 & 25 & \\
Extracranial metastasis & & \\
No & 60 & 0.99 \\
Yes & 67 & \\
Recursive partitioning analysis class & & \\
Class 1 & 100 & 0.13 \\
Class 2 & 40 & \\
Entire cohort & 63 & \\
\hline
\end{tabular}

Bold: Significant values.

Table III. Analysis of freedom from new cerebral lesions.

\begin{tabular}{lcc}
\hline Factor & At 6 months & $p$-Value \\
\hline Age & & \\
$\leq 61$ Years & 100 & 0.92 \\
$>61$ Years & 75 & \\
Gender & & \\
Female & 67 & 0.25 \\
Male & 100 & \\
Number of cerebral lesions & & \\
1 & 80 & 0.75 \\
2 & 100 & \\
Extracranial metastasis & & \\
No & 100 & 0.63 \\
Yes & 67 & \\
Recursive partitioning analysis class & & \\
Class 1 & 100 & 0.92 \\
Class 2 & 86 & \\
Entire cohort & 86 & \\
\hline
\end{tabular}

metastases, the primary tumor is unknown (CUP). Although many studies are available regarding the diagnosis, treatment and prognosis of CUP, in general, there is a lack of data regarding CUP associated with brain metastases (7-11). If a patient presents with multiple cerebral lesions, whole-brain irradiation is the standard treatment (1). In the case of very few brain metastases, local therapies are often used. Neurosurgery is usually limited to a single lesion that is accessible and likely to be removed completely. SRS can be used for a single lesion, particularly if it is not suitable for 
neurosurgical resection, and also for more than one lesion (1). The diameter of each such lesion should not be more than 3-4 cm (1). Currently, there is controversy regarding whether SRS should be administered alone or in combination with whole-brain irradiation.

Two randomized trials have shown that the addition of whole-brain irradiation to SRS resulted in more pronounced neuro-cognitive deficits $(2,3)$. The trial of Chang et al. was stopped by the Data Monitoring Committee after 58 patients, because there was a probability of $96 \%$ that patients receiving SRS plus whole-brain irradiation were significantly more likely to experience a decline in neuro-cognitive function than those receiving SRS alone (52\% versus $24 \%$ at 4 months) (2). In the trial of Brown et al., at the 3-month follow-up, there was less decline in neuro-cognitive function following SRS alone than following SRS plus whole-brain irradiation $(63.5 \%$ versus $91.7 \%, p<0.001$ ) (3). Furthermore, the patients' quality of life was better following SRS alone $(p=0.001)$.

In another randomized trial, omitting whole-brain irradiation resulted in worse intracerebral control $(2,3,11)$. In the trial of Chang et al., the 12-month intracerebral control rates were $27 \%$ after SRS alone and $73 \%$ after SRS plus whole-brain irradiation, respectively $(p<0.001)(2)$. In the trial of Brown et al., the time to intracranial failure was significantly shorter after SRS alone than after the combined approach $(p<0.001)(3)$. In a third randomized trial from Japan, the 12-month intracerebral control rates were $24 \%$ and $53 \%$, respectively $(p<0.001)(11)$. However, in none of the three trials did the improvement in intracerebral control with whole-brain irradiation lead to better survival $(2,3,11)$. Thus, many radiation oncologists do not add whole-brain irradiation to SRS in patients with very few cerebral metastases and adminster SRS alone.

In the present study, the patients received SRS alone for one to two brain metastases from CUP. SRS resulted in excellent local control of the treated lesions; the local control rates at 6 and 12 months were $100 \%$ and $100 \%$, respectively. In addition, the results regarding freedom from new cerebral lesions outside the irradiated areas and regarding survival were promising. The 12-month survival rate of $63 \%$ was similar to the $57 \%$ reported in a previous study of Gamma Knife radiosurgery for brain metastases from CUP (12). In that study, the local control rate was $88.5 \%$, which was slightly lower than in the present series but still promising (12).

In summary, SRS alone showed efficacy with favorable local control and survival rates, and freedom from new brain metastases in patients treated for one to two brain metastases from CUP.

\section{Conflicts of Interest}

On behalf of all Authors, the corresponding Author states that there is no conflict of interest related to this study.

\section{References}

1 Tsao MN, Rades D, Wirth A, Lo SS, Danielson BL, Gaspar LE, Sperduto PW, Vogelbaum MA, Radawski JD, Wang JZ, Gillin MT, Mohideen N, Hahn CA and Chang EL: Radiotherapeutic and surgical management for newly diagnosed brain metastasis(es): An American Society for Radiation Oncology evidence-based guideline. Pract Radiat Oncol 2: 210-225, 2012.

2 Chang EL, Wefel JS, Hess KR, Allen PK, Lang FF, Kornguth DG, Arbuckle RB, Swint JM, Shiu AS, Maor MH and Meyers CA: Neurocognition in patients with brain metastases treated with radiosurgery or radiosurgery plus whole-brain irradiation: a randomised controlled trial. Lancet Oncol 10: 1037-1044, 2009.

3 Brown PD, Jaeckle K, Ballman KV, Farace E, Cerhan JH, Anderson SK, Carrero XW, Barker FG 2nd, Deming R, Burri SH, Ménard C, Chung C, Stieber VW, Pollock BE, Galanis E, Buckner JC and Asher AL: Effect of radiosurgery alone vs radiosurgery with whole-brain radiation therapy on cognitive function in patients with 1 to 3 brain metastases: A randomized clinical trial. JAMA 316: 401-409, 2016.

4 Siegel RL, Miller KD and Jemal A: Cancer statistics, 2017. CA Cancer J Clin 67: 7-30, 2017.

5 Gaspar L, Scott C, Rotman M, Asbell S, Phillips T, Wasserman T, McKenna WG and Byhardt R: Recursive partitioning analysis (RPA) of prognostic factors in three Radiation Therapy Oncology Group (RTOG) brain metastases trials. Int J Radiat Oncol Biol Phys 37: 745-751, 1997.

6 Kaplan EL and Meier P: Non parametric estimation from incomplete observations. J Am Stat Assoc 53: 457-481, 1958.

7 Sivars L, Landin D, Grün N, Vlastos A, Marklund L, Nordemar S, Ramqvist T, Munck-Wikland E, Näsman A and Dalianis T: Validation of human papillomavirus as a favourable prognostic marker and analysis of $\mathrm{CD} 8^{+}$tumour-infiltrating lymphocytes and other biomarkers in cancer of unknown primary in the head and neck region. Anticancer Res 37: 665-673, 2017.

8 Friedrich RE and Zustin J: Mucoepidermoid carcinoma - unknown primary affecting the neck. Anticancer Res 36: 3169-3171, 2016.

9 Sivars L, Tani E, Näsman A, Ramqvist T, Munck-Wikland E and Dalianis T: Human papillomavirus as a diagnostic and prognostic tool in cancer of unknown primary in the head and neck tegion. Anticancer Res 36: 487-493, 2016.

10 Rades D, Conde-Moreno AJ, Garcia R, Veninga T and Schild SE: A tool to estimate survival of elderly patients presenting with metastatic epidural spinal cord compression (MESCC) from cancer of unknown primary. Anticancer Res 35: 6219-6222, 2015.

11 Aoyama H, Shirato H, Tago M, Nakagawa K, Toyoda T, Hatano K, Kenjyo M, Oya N, Hirota S, Shioura H, Kunieda E, Inomata $\mathrm{T}$, Hayakawa K, Katoh $\mathrm{N}$ and Kobashi G: Stereotactic radiosurgery plus whole-brain radiation therapy $v s$. stereotactic radiosurgery alone for treatment of brain metastases. A randomized controlled trial. JAMA 295: 2483-2491, 2006.

12 Niranjan A, Kano H, Khan A, Kim IY, Kondziolka D, Flickinger JC and Lunsford LD: Radiosurgery for brain metastases from unknown primary cancers. Int J Radiat Oncol Biol Phys 77: 1457-1462, 2010.

Received October 30, 2017

Revised November 15, 2017

Accepted November 16, 2017 